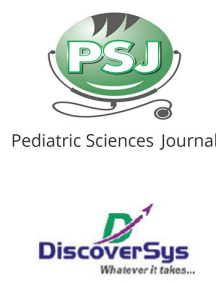

Published by DiscoverSys

\section{Clinical features and neonatal outcomes of neonatus with mother suspected COVID-19 in Malang, Indonesia: a serial-cases, single-centre}

\author{
Brigitta Ida Resita Vebrianti Corebima ${ }^{1 *}$, Eko Sulistijono ${ }^{1}$, Setya Mithra Hartiastuti" \\ Atiek Wulandari ${ }^{2}$, Vanisia Hayu Firdayanti ${ }^{2}$, Nisak Humairoh ${ }^{2}$, Setya Trista Kusumawahyuni ${ }^{2}$
}

${ }^{1}$ Neonatology Division, Child Health Department, Faculty of Medicine, Universitas Brawijaya, Dr. Saiful Anwar General Hospital, Malang, Indonesia ${ }^{2}$ Resident of Child Health Department, Faculty of Medicine, Universitas Brawijaya, Dr. Saiful Anwar General Hospital, Malang, Indonesia

\section{*Corresponding to: Brigitta Ida Resita Vebrianti Corebima; Neonatology Division, Department of Child Health, Faculty of Medicine Brawijaya University, Dr. Saiful Anwar General Hospital, Malang, Indonesia; briqitta vebi@yahoo.com}

Received: 2020-06-02 Accepted: 2020-08-15 Published: 2020-10-25

\section{INTRODUCTION}

The novel coronavirus was first detected in Wuhan City, the capital of Hubei Province, with a population of 11 million. During the first weeks of January 2019, the COVID-19 virus spread rapidly to other countries including Thailand, Japan, Korea, United States, and Iran. On 7 January 2020 scientists in China identified the etiological agent of the epidemic as a previously unknown coronavirus, and it was given the designation 2019-nCoV (for 2019 novel coronavirus). On February 11, 2020, the novel coronavirus disease received an official name by the World Health Organization (WHO), Coronavirus Disease 19 (COVID-19) and taxonomy of Viruses has proposed SARS-CoV-2 as the name of the virus that causes COVID-19. The most common manifestations of COVID-19 consist of fever, cough, and fatigue or myalgia, sputum production, and headache. ${ }^{1}$

As COVID-19 virus is still spreading, more infections in pregnant women are likely to be seen. Whether COVID-19 increases the risk of miscarriage, stillbirth, preterm delivery, fetal tachycardia and fetal distress is unknown. ${ }^{1}$ The COVID-19 pandemic has affected every facet of life, beginning with the first hours and days after from the hospital after two days of isolation in the hospital with negative Nucleic Acid Test of SARS-CoV-2 results.

Conclusion: The clinical characteristics of neonates and neonatal outcome appear very good, and these outcomes are achieved with intensive, active management, which might be the best practice in resuscitation, stabilization, bathing the baby before going to the isolation ward, and management the patient in the isolation ward

Keywords: Clinical Features, Neonatal, Outcomes, COVID-19

Cite this Article: Corebima, B.I.R.V., Sulistijono, E., Hartiastuti, S,M., Wulandari, A., Firdayanti, V,H., Humairoh, N., Kusumawahyuni, S.T. 2020. The correlation of 25-Hydroxyvitamin D (25-(OH)D) to lgE level, eosinophil, and Asthma Control Test (ACT) score in asthma patients. Pediatrics Sciences Journal 1(1) (Supplementary): 27-34.

birth, some have argued that the prudent course is to isolate infants from mothers with suspected or confirmed COVID-19 in order to reduce the risk of transmission from mother to baby. But data are limited, and recommendations for the first days after birth differ. The World Health Organization recommends that infants and mothers with suspected or confirmed COVID-19 "should be enabled to remain together and practice skin-toskin contact, kangaroo care and to remain together and to practice rooming in throughout the day and night." Breastfeeding is strongly recommended given its known lifelong importance for maternal and child health. Mothers are encouraged to wash their hands, wear a mask if they have a cough, and routinely disinfect surfaces that they have touched. The causative agent of this unexplained infected pneumonia was identified as severe acute respiratory syndrome coronavirus 2 (SARS-CoV-2) which not only has a strong human-to-human transmission but also causes severe pneumonia to death. ${ }^{2}$

Severe acute respiratory syndrome coronavirus 2 (SARS-Cov-2) is so aggressive that the infection has been transmitted to other countries and is seriously imperiling human life. World Health Organization has declared this disease to constitute a Public 
Health Emergency of International Concern on 30 January $2020 .^{3}$ A total of 26.359 suspected cases and 31225 confirmed cases with 639 deaths linked to this pathogen on 7 February 2020. Different age group are generally susceptible to SARS-CoV-2. ${ }^{3}$ Neonatologists should be vigilant assessing newborn babies delivered by infected mother or brought up by infected house maid and improve the knowledge of prevention and treatment of COVID-19.

As per the Centers for Disease Control and Prevention (CDC), it is not known if pregnant mothers are at increased risk of acquiring SARSCoV-2 infection or whether they are at more risk for developing serious illness as a result. ${ }^{4}$ It remains unclear if vertical transmission of the virus occurs during pregnancy. Limited evidence suggests that vertical transmission in utero is rare including a report of nine pregnant infected women in China whose infants all tested negative for the virus. ${ }^{5}$

However, more recently, immunoglobulin (Ig)-M antibodies have been detected in newly born infants, although viral RNA has not been isolated, suggesting a possibility of vertical transmission. In addition, disruption of the placental barrier in placental abruption or maternal-fetal hemorrhage may potentially transmit the virus and/or IgM antibodies to the fetal circulation. Based on our knowledge of other viruses that cause severe respiratory illness (influenza, SARS-CoV, and Middle East Respiratory Syndrome Coronavirus [MERSCoV]), neonates, especially those born preterm, are at increased risk of acquiring COVID-19 postnatally secondary to an immature immune system. Resuscitating a neonate born to a mother suspected or confirmed to have COVID-19 poses a risk to the neonate and health care providers. With limited availability of literature to guide perinatal management, the intent of this manuscript is to provide a stepwise approach to resuscitating, stabilizing, and providing postnatal care to an infant born to a mother who is suspected or has confirmed COVID-19. As more evidence becomes available, these guidelines are likely to evolve. $^{6}$

\section{CASE PRESENTATION 1}

The mother of the neonatal patient is a 40 -year-old pregnant woman who lives in a city near Malang. She has no contact with confirmed or suspected cases of SARS-CoV-2 during pregnancy and her family has no confirmed or suspected cases of SARS-CoV-2, but the city is the one of the red zone areas in East Java. She had no history of hypertension, diabetes, or heart disease. On 30 April 2020, the pregnant woman (at 34-36 weeks' gestation) with partus prematourus imminents, reccurent fluxus and rapid test of SARS-CoV-2 was positif. She had no history of fever, cough, swallowing pain, and dispneu before delivery. Fetal heart rate was $136 \mathrm{bpm}$ and fetal heart monitoring showed no abnormality. Emergency cesarean delivery was performed. At $11.00 \mathrm{am}$, she delivered a baby girl weighing $2100 \mathrm{~g}$. Apgar scores at 1 and 5 minutes were 4 and 6, with muscle tone weak but crying was loud, during observation after delivery the baby down score was 1 with respiratory rate 64 times/ minute. We have done the standart resuscitation and stabilitation in separate room with the mother, and gave bath after the baby was stable. The mother had been wearing an N95 mask throughout the operation, and the baby had no contact with the mother after birth. The infant and her mother were transferred to the PINERE (covid-19 room isolation) 10 minutes after birth for close observation and rooming in but the baby in the incubator, and we give expressed breast milk.

Blood tests of the newborn revealed the normal limited, with white blood cell $20.780 / \mu \mathrm{L}$, haemoglobine $13,6 \mathrm{~g} / \mathrm{dL}$, haemathocryte $38,4 \%$, platelete $233.000 / \mu \mathrm{L}$, eosinophil $0,9 \%$, basophil 0,3 $\%$, neutrophil $64,7 \%$, lymphosite $26,4 \%$ monocyte $7,7 \%$, procalcitonin 0,21 , CRP 0,02 , IT ratio 0,07 , rapid test of SARS-CoV-2 was negative, the result of first and second The result of pharyngeal swab for SARS-CoV-2 was negative. The chest radiograph of the neonate, obtained on 30 April 2020, no abnormalities in the lung and heart.

On $1^{\text {st }}$ may 2020, the second day after the surgery, the newborn vital signs were stable with the blood oxygen saturation maintained > $95 \%$ with $0,5 \mathrm{lpm}$ oxygen nasal canule with the downscore 1 , the respiratory rate was 62 times per minute, her pulse was 130 beats per minute with strong and regular pulse and temperature of 36.6 degrees celcius. Patient had got treatment antibiotic ampicillin sulbactam intravenouse $200 \mathrm{mg} / \mathrm{kgBW} /$ days, gentamisin intravenouse $5 \mathrm{mg} / \mathrm{kgBW} /$ days, aminofilin intravenous $6 \mathrm{mg} / \mathrm{kgBW} /$ days.

On $2^{\text {nd }}$ may 2020,the vital signs of newborn was stable, with air oxygen the oxygen saturation can maintained $>95 \%$ with downscore was 0 , her respiratory rate was 52 times per minute, her pulse was 140 beats per minute with strong and regular pulse and temperature of 36.7 degrees celcius.

On $3^{\text {th }}$ may 2020, the newborn haemodinamic was stable, absence of complaint or discomfort like apnea or vomiting. The newborn and mother have discharge from the hospital, and continue to home isolation.

\section{CASE PRESENTATION 2}

Mother of the neonatal patient is a 31-year-old 


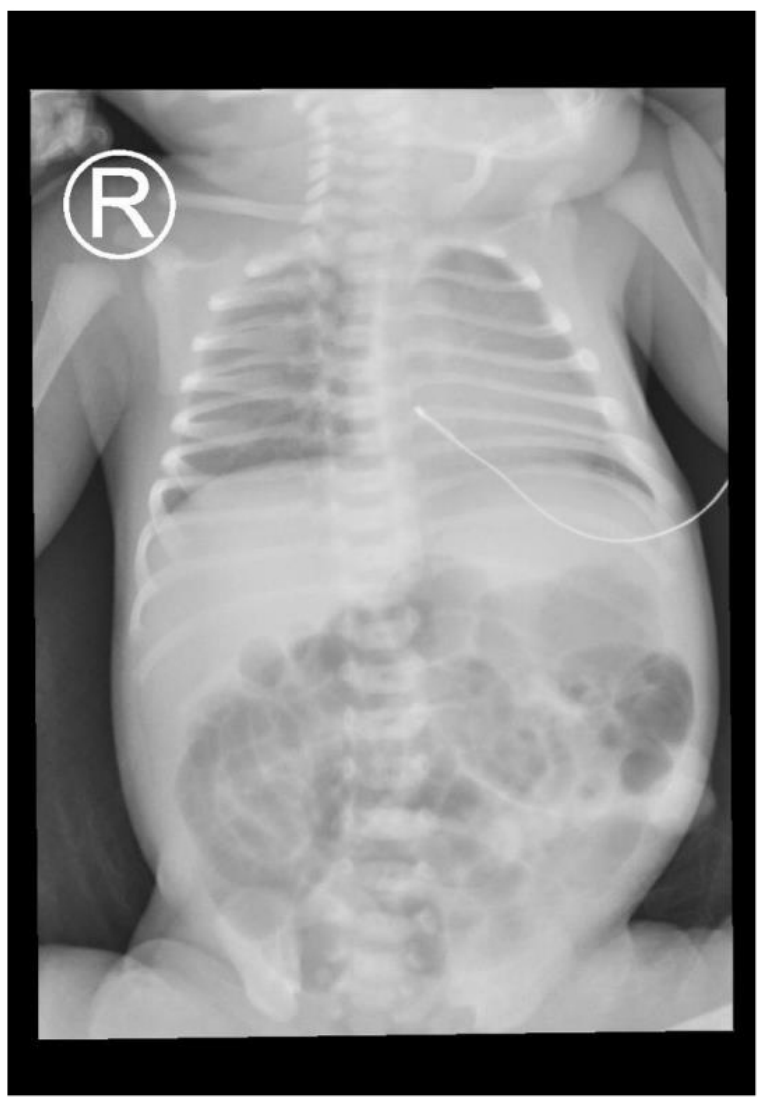

Figure 1. Chest radiograph of the newborn, obtained on 30 April 2020, showing no abnormalities in the lung and heart.

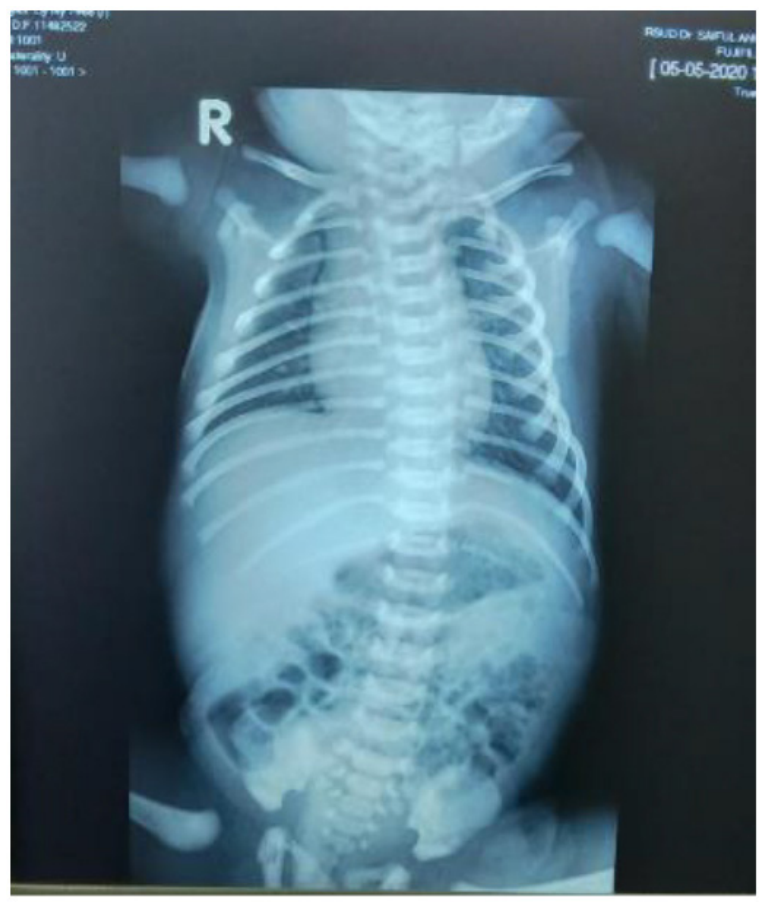

Figure 2. Chest radiograph of the newborn, obtained on May 5th 2020, showing no abnormalities in the lung and heart. pregnant woman who lives in a city near Malang. She was referred by the general physician with G2P1001Ab000 39/40 weeks and suspected for COVID-19. The mother was working in the hospital in Malang where she got the rapid test and showed positive result. The mother of the patient complained for fever and went to the doctor on May $4^{\text {th }} 2020$, due to the history of positive for Cov-2 rapid test, the physician decided to refer the patient to Saiful Anwar general Hospital. She has no contact with confirmed or suspected cases of SARS-CoV-2 during pregnancy and her family has no confirmed or suspected cases of SARS-CoV-2.

However she works as a pharmacist in the hospital in Malang and got the rapid test on April $29^{\text {th }}$ 2020. She had no history of hypertension, diabetes, or heart disease. On May 4th 2020, the pregnant woman (at 39-40 weeks' gestation) with febrile day 1 , fetal tachycardia, myopia and suspected COVID-19. She had no history of fever, cough, swallowing pain, and dispneu before delivery. Fetal heart rate was $136 \mathrm{bpm}$ and fetal heart monitoring showed no abnormality.

Emergency cesarean delivery was performed. At $11.00 \mathrm{am}$, she delivered a baby girl weighing 2900 g. Apgar scores at 1 and 5 minutes were 7 and 9, with strong muscle tone but crying was loud, during observation after delivery the baby down score was 1 with respiratory rate 54 times/ minute. We have done the standart resuscitation and stabilitation, and gave bath after the baby was stable. The mother had been wearing an N95 mask throughout the operation, and the baby had no contact with the mother after birth. The infant and her mother were transferred to the PINERE (covid-19 room isolation) 10 minutes after birth for close observation and rooming in but the baby in the incubator, and we give expressed breast milk.

\section{CASE PRESENTATION 3}

The mother of the neonatal patient is a 28 -year-old pregnant woman who lives in a city near Malang. She has no contact with confirmed or suspected cases of SARS-CoV-2 during pregnancy and her family has no confirmed or suspected cases of SARS$\mathrm{CoV}-2$, but the city is the one of the red zone areas in East Java. She had no history of hypertension, diabetes, or heart disease. On May $3^{\text {rd }} 2020$, the pregnant woman (at 38-39 weeks' gestation) was consulted with reactive HBsAg antibody and rapid test of SARS-CoV-2 was positive. She had no history of fever, cough, swallowing pain, and shortness of breath prior to delivery. Fetal heart rate was $140 \mathrm{bpm}$ and fetal heart monitoring showed no abnormality. Caesarian section was performed on May $3^{\text {rd }} 2020$ at 4.00 p.m., she delivered a baby 


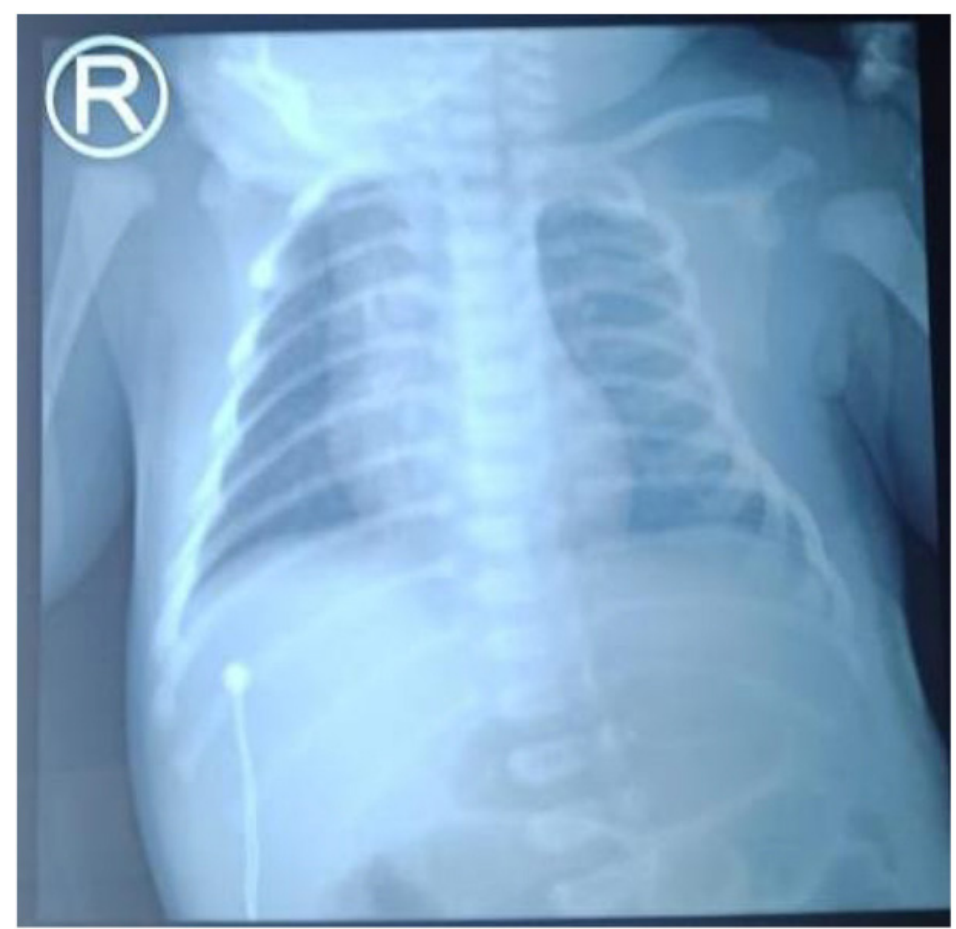

Figure 3. Chest radiograph of the newborn, obtained on May $3^{\text {rd }}$ 2020, showing no abnormalities in the lung and heart.

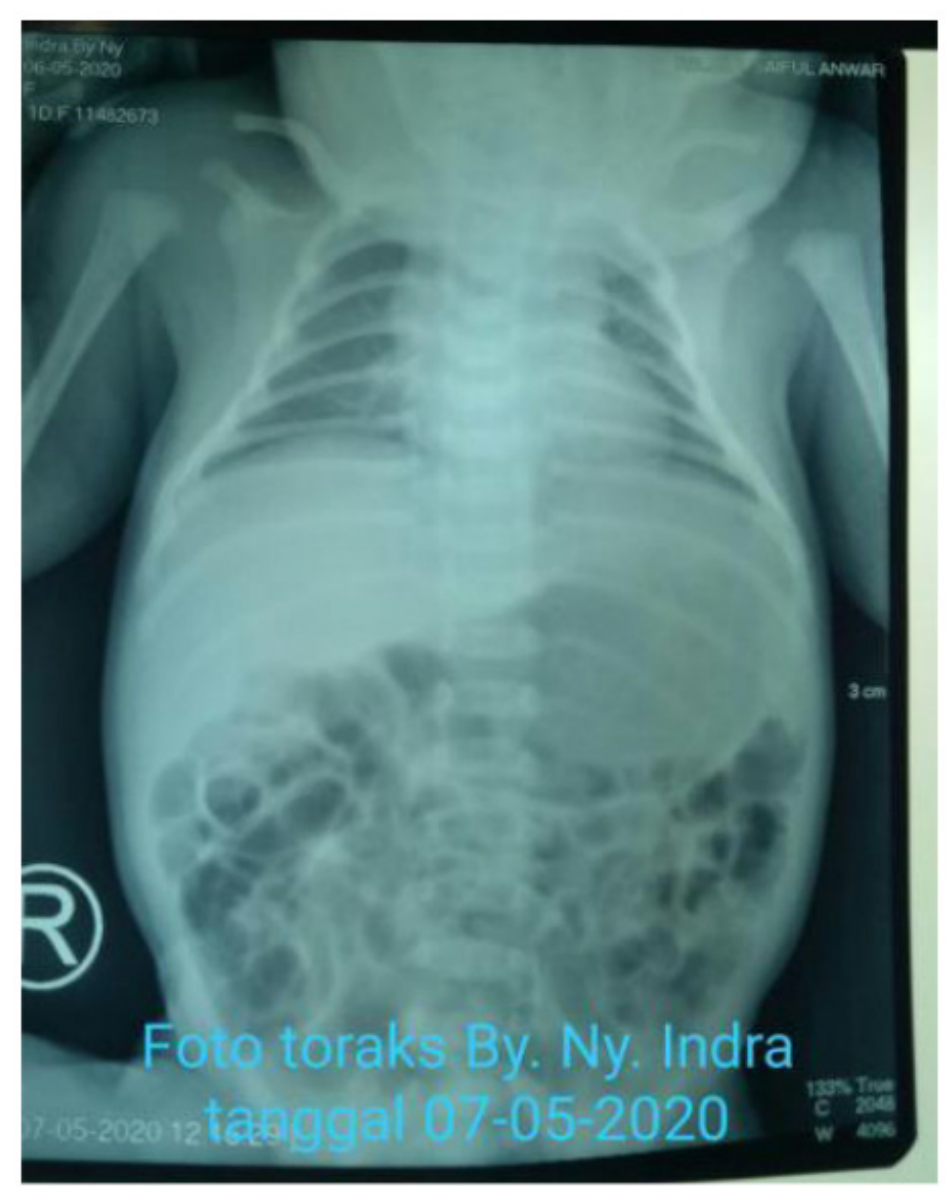

Figure 4. The patient's chest X-Ray girl weighing 3300 grams. Apgar scores at 1 and 5 minutes were 6 and 8 , with muscle tone weak but crying was loud, during observation after delivery the baby's downe score was 0 with respiratory rate 40 times/ minute. We have done the standart resuscitation and stabilitation in separate room with the mother, and gave bath after the baby was stable. The mother had been wearing an N95 mask throughout the operation, and the baby had no contact with the mother after birth. The infant and her mother were transferred to the PINERE (covid-19 room isolation) 10 minutes after birth for close observation and rooming in but the baby in the incubator, and we give expressed breast milk.

Blood tests of the newborn revealed the normal limited, with white blood cell $17640 / \mu \mathrm{L}$, haemoglobine $16.1 \mathrm{~g} / \mathrm{dL}$, haemathocryte $46.4 \%$, platelete $312000 / \mu \mathrm{L}$, eosinophil $0.6 \%$, basophil 0,4 $\%$, neutrophil $74.8 \%$, lymphosite $15.5 \%$ monocyte $8.8 \%$, procalcitonin 0.14 , CRP 0.01 , IT ratio 0.03 , rapid test of SARS-CoV-2 was negative, the result of first and second The result of pharyngeal swab for SARS-CoV-2 was negative. The chest radiograph of the neonate, obtained on May $3^{\text {rd }} 2020$, no abnormalities in the lung and heart.

On May $4^{\text {th }} 2020$, the second day after the delivery, the newborn vital signs were stable with the blood oxygen saturation maintained $>95 \%$ with ambient oxygen,the respiratory rate was 54 times per minute, her pulse was 136 beats per minute with strong and regular pulse and temperature of 36.6 degrees celcius. Patient had got treatment antibiotic ampicillin sulbactam intravenouse $200 \mathrm{mg} / \mathrm{kgBW} /$ day, gentamisin intravenouse $5 \mathrm{mg} / \mathrm{kgBW} /$ day for three days.

On May $5^{\text {th }} 2020$,the vital signs of newborn was stable, with ambient oxygen the oxygen saturation can achieve $>95 \%$ with downe score was 0 , her respiratory rate was 52 times per minute, her pulse was 145 beats per minute with strong and regular pulse and temperature of 36.7 degrees celcius. The newborn and mother was discharged from the hospital, and continue to home isolation for the next 14 days.

\section{CASE PRESENTATION 4}

The fourth patient was referred from Malang public regional hospital. She lives in Malang city, Indonesia. Neither She nor her family has contact with confirmed or suspected cases of SARS-CoV-2. she is nulligravida. a woman who has delivered her first baby on May 6, 2020 in Saiful Anwar Hospital. She is referred from Malang public regional hospital by the general physician with G1P0000Ab000 38-39 weeks and suspected for COVID-19. the mother's with a history of rapid test of $\operatorname{IgM}+$ covid in April 
29,2020 . She works as administrative medical of Malang public regional hospital. She told that she haven't symptoms like fever, cough, or shortness of breath. There wasn't abnormal condition during her pregnancy, she haven't get fluxus or vaginal bleeding, no fever with rash in Trimester I, No asthma, No Hypertension, no Diabetes mellitus, no Exposure to smoke. The mother routine control in midwives. She also had examined with Ultrasonography with a conclusion within normal limit. The baby started to cry immediately after birth, with good muscle tone, and clear amniotic fluid. The doctor and tim start the initial steps of resuscitation Heart Rate 100 bpm, Respiratory Rate 50 tpm, with saturation $88 \%$ before delivery. We have done the standart resuscitation and stabilitation, and gave bath after the baby was stable.

The newborn vital signs were stable with the blood oxygen saturation maintained $97 \%$ with room oxygen with the Downe score zero, the respiratory rate was 52 times per minute, her pulse was 158 beats per minute with strong and regular pulse and temperature of 36.8 degrees celcius.

Blood tests of the newborn was obtained on May $7^{\text {th }}$ and revealed the normal limit. White blood cell $21.660 / \mu \mathrm{L}$, haemoglobin $14.8 \mathrm{~g} / \mathrm{dL}$, haematocrit $40,1 \%$, platelet $297.000 / \mu \mathrm{L}$, eosinophil $0,0 \%$, basophil 0,4 \%, neutrophil 79,8 \%, lymphocyte 11,1 $\%$ monocyte $8,7 \%$, procalcitonin 2,17 , CRP 0,06 , IT ratio 0,6 , The result of pharyngeal swab for SARS$\mathrm{CoV}-2$ is waiting for result. The chest radiograph of the neonate, obtained on May $17^{\text {th }}, 2020$. The chest Xray showed within normal limit.

Our patients were diagnosed as aterm and asymptomatic Covid-19. Patients were given oxygen room therapy, intramuscular injection of hepatitis B0 $0.5 \mathrm{cc}$, intramuscular injection of vitamin $\mathrm{K} 1$ $\mathrm{mg}$, administration of eye ointment for the right and left eye Chloramphenicol. Thermoregulation of 36.5 to 37.5 is performed, and with full feeding. All of the patient's summary can be seen on Table 1 .

\section{DISCUSSION}

As COVID-19 virus is still spreading, more infections in pregnant women are likely to be seen. Whether COVID-19 increases the risk of miscarriage, stillbirth, preterm delivery, fetal tachycardia and fetal distress is unknown. According to the official website of Ministry of Health and Medical Education (MOHME) in Mazandaran and Zanjan provinces, Iran, three infants were born from infected pregnant mothers. Among these three cases of COVID-19 infection there were two mothers who developed acute respiratory distress syndrome (ARDS) after delivery and died. According to the websites, their neonates were negative when tested for COVID-19. ${ }^{7}$ It seems that COVID-19 infection in pregnant patients may increase the risk of maternal mortality. Chen et al., reported the clinical characteristics and placental pathology from three women with confirmed COVID-19 infection. No COVID-19 nucleic acid was detected in the placentas or neonatal throat swabs by RT-PCR. Moreover, they found that the clinical characteristics of infected pregnant women in late pregnancy were similar to those of nonpregnant patients, and no severe adverse pregnancy outcome was found in the three cases. ${ }^{8}$

In other study, Chen et al., evaluated clinical

Table 1. Patient Characteristic Summary

\begin{tabular}{|c|c|c|c|c|}
\hline & Patient 1 & Patient 2 & Patient 3 & Patient 4 \\
\hline Pregnancy Outcome & Discharged & Discharged & Discharged & Discharged \\
\hline Neonatal Outcome & Normal & Normal & Normal & Normal \\
\hline \multicolumn{5}{|l|}{ Birthweight } \\
\hline Apgar Score (1 min) & 7 & 7 & 7 & 7 \\
\hline Apgar Score (5mins) & 9 & 9 & 9 & 9 \\
\hline Admission to PINERE & Yes & Yes & Yes & Yes \\
\hline Nucleic Acid Test of SARS-CoV-2 & Negative & Negative & Negative & Negative \\
\hline \multirow[t]{2}{*}{ Days of Follow Up } & 2 days in hospital & 2 days in hospital & 2 days in hospital & 2 days in hospital \\
\hline & 14 days by phone & 14 days by phone & 14 days by phone & 14 days by phone \\
\hline Neonatal Complications & No & No & No & No \\
\hline
\end{tabular}


records, laboratory results, and chest computed tomography (CT) scans among nine pregnant women with laboratory-confirmed COVID-19 who were referred to Zhongnan Hospital of Wuhan University, Wuhan, China, between January 20 and January 31, 2020. Their results revealed that the clinical characteristics of COVID-19 in pregnant women were similar to infected non-pregnant women. They reported nine livebirths and none of the patients developed severe COVID-19 or died, and all samples tested negative for the virus in neonates. Their findings supported that there is currently no sevidence for vertical transmission in infected mothers in late pregnancy. ${ }^{5}$

In Saiful Anwar hospital, Malang, there are four cases of COVID-19 in pregnant women, in three case there were no clinical signs, only one case had a fetal tachycardia. In Saiful Anwar Hospital, Malang, all the pregnant women have a test of SARS-CoV-2 before delivery, but only rapid test, for pharyngeal swab for SARS-CoV-2 will performed if the rapid test SARS-CoV-2 positive, and the swab result will be finished after one week. All case of pregnant women have a positive of rapid test SARS-CoV-2 but all the result of rapid test and pharyngeal swab SARS-CoV-2 in newborn were negative. This result appropriate to study of chen et al, there are no evidence for vertical transmission in infected mothers in pregnancy.

In Saiful Anwar Hospital, Malang, testing capacity is available, neonates should be tested for SARS CoV- 2 infection using available molecular assays. Testing well newborns may facilitate plans for care after birth hospital discharge, and will contribute to the overall understanding of viral transmission. Testing infants who require prolonged neonatal intensive care should be performed to determine the potential contribution of COVID-19 to observed clinical illness. The optimal timing and extent of testing is currently unknown. Informed by limited data on viral detection, and with the aim of distinguishing transient viral colonization from established infection. The testing procedures in our hospital consider using swabs of nasopharyngeal and oropharyngeal at 24 and 48 hours after birth (six swabs). One swab that samples first the oropharyngeal and then the nasopharynx may be used to conserve swabs and PCR testing reagents. This single swab then should be placed in one viral transport media tube and sent to the lab for a single PCR test to be performed.

The resuscitation delivery of the newborn from pregnant woman with COVID-19, we use the AAP and CDC recommendation. The process of delivery and resuscitation in negative pressure room. No visitors/partners allowed, video link only, for droplet and contact precautions we use PAPR or N95 masks + eye shield gown and glove, and the mother use mask. Neonatal resuscitation and further care in a separate room followed by warm water bath to remove virus potentially present on skin surfaces, if the baby's stable, and the placement in an isolette. No delayed cord clamping under any circumstance and no skin to skin contact. Additional personnel may be outside the room with the incubator transport isolette and be available if extensive neonatal resuscitation is needed. In Saiful Anwar Hospital, Malang, the newborn was transferred to COVID-19 isolette room with the mother after delivery.

Further management, the newborn then transferred to the isolation ward through separated hallway in the hospital. The team accompanying the newborn wear standardized personal protection equipment (level 4). The transport process for the neonate was done effectively without having to wait for the patient's mother to finish through all the processes after delivery. In the other words, the newborn will be transferred to the isolation room at the earliest time. The newborn baby thus transferred into the COVID-19 ward in a special room designated for the newborn and the mother. The room is equipped by incubator for the newborn and a HEPA filter. The HEPA filter is designed to arrest very fine particles effectively. The newborn should be maintained at least 6 feet apart and/or placed in air temperature-controlled isolation for cohorting. ${ }^{9}$

Saiful Anwar General Hospital adheres to the mother-centered care which allows the neonate to be treated in the similar ward with the mother. Certainly, with several conditions, both mother and newborn baby are both must be in good condition, do not require special oxygen management such as the use of CPAP or ventilators. However, if intensive care is needed for either the mother or the baby, separate care is necessary.

Furthermore, to support the diagnosis, at Saiful Anwar Hospital, an oropharyngeal swab is performed on the baby. Swabs are performed on infants with mothers whose nasopharyngeal swabs are positive. None of the four patients and the mothers are positive until now. However, the swabs will be done two times within 24 hours using specimen from the infants' nasopharyng and oropharyng.

No study to date has demonstrated the presence of SARS-CoV-2 in breast milk. Mothers may express breast milk (after appropriate breast and hand hygiene) and this milk may be fed to the infant by designated caregivers. Breast pumps and components should be thoroughly cleaned in between pumping sessions using standard center 
policies that must include cleaning the pump with disinfectant wipes and washing pump attachments with hot soapy water. In addition to the known benefits of breastfeeding, mothers' milk may provide infant protective factors after maternal COVID-19. Promoting breast milk feeding and supporting establishment of maternal milk supply may offer additional benefits to well and sick newborns. ${ }^{9}$

In Saiful Anwar General Hospital, expressed breast milk for the infant is recommended. The hygiene method applied to the mother should become special consideration. The breast should be thoroughly cleaned between pumping sessions. Cleaning pump tool using disinfectant wipes and washing pump attachments with warm soapy water are highly recommended and mother should use mask before the process. The use of milk donor is not recommended.

Infants determined to be infected by molecular testing (or whose status cannot be determined due to lack of testing), but with no symptoms of COVID-19, may be discharged home on a case-bycase basis with appropriate precautions and plans for frequent outpatient follow-up contacts (either by phone, telemedicine, or in-office) through 14 days after birth. Specific guidance regarding use of standard procedural masks, gloves and hand hygiene should be provided to all caretakers. Infants with negative SARS-CoV-2 molecular testing should optimally be discharged to the care of a designated healthy (non-infected) caregiver. If the mother is in the same household, she should maintain a distance of at least 6 feet for as much of the time as possible, and when in closer proximity to the neonate should use a mask and handhygiene for home newborn care until either (a) she has been afebrile for 72 hours without use of antipyretics, and (b) at least 7 days have passed since symptoms first appeared; or she has negative results of a molecular assay for detection of SARS-CoV-2 from at least two consecutive nasopharyngeal swab specimens collected $\geq 24$ hours apart. ${ }^{9}$

In this hospital the newborn observed until 48 hours, if the newborn and mother have no manifestation of COVID-19 and have a negative result from rapid test COVID-19, they can be discharged and continue to observation in their home. During the pandemic, obstacles found in determining the case of confirmation because of the limited screening tools in Indonesia. Nevertheless, both the mother and the baby will be followed up by local health service even after hospital discharge. The post-hospital discharge management will be done comprehensively starting from the process of picking up patients to follow-up at home using the questionnaire method.

\section{CONCLUSION}

In conclusion, the clinical characteristics of neonates and neonatal outcome appear very good, and these outcomes are achieved with intensive, active management, which might be the best practice in resuscitation, stabilization, bathing the baby before go to isolation ward, and management in patient in isolation ward. If the mother has suspected covid, both are stable, and the infant is not preterm, neonatal health care providers must offer orientations regarding precautions to avoid spreading the virus, including washing of the mother's hands before touching the infant, using a face mask while breastpumping, and staying inisolated rooming-in. Breastfeeding is strongly recommended, given its known lifelong importance for maternal and child health. Mothers are encouraged to wash their hands, wear a mask if they have a cough, and routinely disinfect surfaces that they have touched.

\section{CONFLICT OF INTEREST}

The Authors declare no conflict of interest

\section{FUNDING}

This research was funded privately by authors

\section{ETHICAL CLEAREANCE}

This research was conducted after obtaining approval from the ethics commission RSAA Malang Research.

\section{AUTHOR CONTRIBUTION}

All authors contributed to the process of this research

\section{REFERENCE}

1. Mojgan Karimi-Zarchia, Hossein Neamatzadeh, Seyed Alireza Dastgheib, Hajar Abbasi, Seyed Reza Mirjalilid, Athena Behforouz, Farzad Ferdosiang, and Reza Bahramii. 2020. Vertical Transmission of Coronavirus Disease 19 (COVID-19) from Infected Pregnant Mothers to Neonates: A Review. Fetal and pediatric pathology. https://doi.org/10 .1080/15513815.2020.1747120.

2. Wang C, Horby PW, Hayden FG, Gao GF. 2020. A novel coronavirus outbreak of global health concern. Lancet. ; 395(10223):470-473.

3. Huang C, Wang Y, Li X, et al. 2020. Clinical features of patients infected with 2019 novel coronavirus in Wuhan, China. Lancet. 2020;395(10223):497-506.

4. Centers for Diasase Control and Presention Pregnancy \& breastfeeding information about coronavirus disease. 2019. Available at: https://www.cdc.gov/coronavirus/2019-ncov/ prepare/pregnancy-breastfeeding.html. Accessed April 2, 2020 
5. Chen H, Guo J, Wang C, et al. 2020. Clinical characteristics and intrauterine vertical transmission potential of COVID-19 infection in nine pregnant women: a retrospective review of medical records. Lancet ;395(10226):809-815

6. Praveen Chandra sekharan, Maximo Vento, Daniele Trevisanuto, et al. 2020. Neonatal Resuscitation and Postresuscitation Care of Infants Born to Mothers with Suspected or Confirmed SARS-CoV-2 Infection. Am J Perinatol. DOI https://doi.org/ 10.1055/s-0040-1709688.

7. Tasnim Agency. 2020. Birth of a neonate from infected mother COVID-19 in Babol city; Mar 3.

8. Chen S, Huang B, Luo DJ, Li X, Yang F, Zhao Y, Nie X, Huang BX. 2020. Pregnant women with new coronavirus infection: a clinical characteristics and placental pathological analysis of three cases. Zhonghua Bing Li Xue Za Zhi. ;49:E005. doi:10.3760/cma.j. cn112151-2020022500138.

9. Karen M. Puopolo, Mark L. Hudak, David W. Kimberlin, James Cummings .2020. Management of Infants Born to Mothers with COVID-19 Date of Document: April 2, 2020. American Academy of Pediatrics Committee on Fetus and Newborn, Section on Neonatal Perinatal Medicine, and Committee on Infectious Diseases.

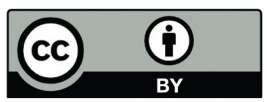

This work is licensed under a Creative Commons Attribution 